\title{
Linear response theory for open quantum systems within the framework of the ABL formalism
}

\author{
Masashi Ban
}

Received: 15 December 2014 / Accepted: 13 February 2015 / Published online: 24 February 2015

(C) Chapman University 2015

\begin{abstract}
A linear response theory of the strong and weak values in open quantum systems is developed within the framework of the Aharonov-Bergmann-Lebowitz formalism of quantum mechanics. The measurement of an observable influenced by an external field is performed between pre-selection and post-selection. The system irreversibly evolves from a pre-selected state to a post-selected one due to an interaction with a surrounding environment. The effect of the external field on the measured observable is described in terms of forward and backward response functions. A backward response function appears in the theory since the post-selection of the system is performed. Furthermore, the results are extended beyond the strong and weak values.
\end{abstract}

Keywords Linear response $\cdot$ Strong and weak values $\cdot$ Post-selection $\cdot$ Open quantum system

\section{Introduction}

Quantum measurement [1-3] plays an essential role in quantum mechanics, which is indispensable for extracting information from a physical system of interest. It irreversibly and probabilistically changes a quantum state of a measured system. In a physical process, a system is first prepared in an initial state (a pre-selected state) and it evolves with time until measurement is performed on the system. Measurement causes the state reduction and after that the system re-starts to evolve with time. Usually, the system after the measurement is irrelevant since the measurement provides the information on the system that we need. It should be noted that although the measurement result is affected by the system state before the measurement, it is not affected by that after the measurement. Hence, the theory is not symmetric before and after the measurement. However, Aharonov, Bergmann and Lebowitz (ABL) [4] have shown in their seminal paper that the time symmetric formulation of quantum mechanics is possible by introducing post-selection of a quantum system after measurement into the theory. In their formulation, the system after the measurement evolves with time until post-selection is done at final time. In other word, quantum states of the system are fixed at initial and final time, while in the usual formulation, only an initial state is fixed. In the $\mathrm{ABL}$ formalism, the symmetry can be found if measurement on the system is performed between pre-selection and post-selection. Aharonov and Vaidman [5] have extended the theory so as to treat the case that a post-selected state is correlated with a pre-selected one, and they have also developed the two-state vector formalism [6-8] to

M. Ban $(\varangle)$

Graduate School of Humanities and Sciences, Ochanomizu University, 2-1-1 Ohtsuka, Bunkyo-ku, Tokyo 112-8610, Japan

e-mail: ban.masashi@ocha.ac.jp 
systematically describe quantum systems with pre- and post-selection. Furthermore, the weak value of an observable [9] has been found within the framework of the ABL formalism. The weak value has been attracted much attention and studied extensively due to the peculiarity and the potential applicability [10-22].

The linear response theory developed by Kubo [23] provides a useful method for investigating properties of physical systems in many research fields, which has been applied to various kinds of phenomena from solid-state physics to quantum optics [23-26]. In the linear response theory, applying a weak external field, we calculate the change of an average value of a system observable up to the first order with respect to the applied field. The linear response theory provides microscopic expressions of various parameters characterizing physical systems such as conductivity and susceptibility [23,24]. The linear response of the average value depends only on the external field applied before the measurement is performed. The external field applied after the measurement of an observable never affects the measurement outcome. In this sense, we can say that the linear response theory is not symmetric. However, when post-selection is performed on the system, the linear response is affected by the external field applied after as well as before the measurement [27]. Hence, it may be expected that some symmetric property can be found in the linear response if it is formulated within the framework of the ABL formalism.

In this paper, using on the ABL formalism [4], we formulate the linear response theory [23] for the strong and weak values to an external field, where a quantum system is influenced by a surrounding environment [28]. The preliminary result has been reported in Ref. [27]. In Sect. 2, we provide expressions of the strong and weak values of an observable, where the measurement is performed on the system between pre-selection and post-selection. Then, the system irreversibly evolves with time from a pre-selected state to a post-selected one under the influence of an environment. Since the Markovian property (the semi-group property) is assumed, the time evolution is described by the quantum master equation of the Lindblad form [28-30]. In Sect. 3, we investigate the linear response of the strong and weak values to an external field. The forward and backward response functions are derived, which characterize the linear response of the system. The former corresponds to the usual response function in the absence of the post-selection. The backward response function describes the effect of the external field applied after the measurement of the observable, which is a result of the post-selection of the system. In Sect. 4, using the method provided in Ref. [31], we extend the results beyond the strong and weak values of an observable. In Sect. 5, we provided a brief summary of this paper.

\section{Strong and weak values of an open system with post-selection}

In this section, we review the strong and weak values of an observable of an open quantum system which interacts with its surrounding environment. Measurement of a system observable is performed at intermediate time between preselection and post-selection of the system. Time evolution of the system becomes irreversible due to the interaction with the environment [28-30]. The irreversibility of the time evolution significantly affects the strong and weak values of the measured observable [32].

We suppose that a quantum system to be measured is prepared in a quantum state $\hat{\rho}_{i}$ at initial time $t_{i}$. The whole state of the system and environment is given by $\hat{W}_{i}=\hat{\rho}_{i} \otimes \hat{\rho}_{E}$ with an environmental state $\hat{\rho}_{E}$ which is usually assumed to be a thermal equilibrium state. In this paper, we consider the case that there is no initial correlation between the system and the environment. The system and environment evolve into $\hat{W}\left(t_{m}\right)=\hat{U}\left(t_{m}, t_{i}\right) \hat{W}_{i} \hat{U}^{\dagger}\left(t_{m}, t_{i}\right)$ just before the measurement is performed at time $t_{m}$, where $\hat{U}\left(t, t^{\prime}\right)$ is a timeevolution unitary operator of the system and environment. Measurement of a system observable $\hat{A}$ at time $t_{m}$ changes the state as $\hat{W}\left(t_{m}\right) \rightarrow \hat{W}\left(t_{m} ; a\right) / \operatorname{Tr} \operatorname{Tr}_{E} \hat{W}\left(t_{m} ; a\right)$, where the non-normalized post-measurement state $\hat{W}\left(t_{m} ; a\right)$ depends on the measurement outcome $a$ and $\operatorname{Tr}\left(\operatorname{Tr}_{E}\right)$ stands for the trace operation over a Hilbert space of the system (the environment). After performing the measurement, the system and environment evolve again with time. The quantum state just before the post-selection of the system at time $t_{f}$ becomes $\hat{W}\left(t_{f} ; a\right) / \operatorname{Tr} \operatorname{Tr}_{E} \hat{W}\left(t_{f} ; a\right)$, where the non-normalized state $\hat{W}\left(t_{f} ; a\right)$ is given by $\hat{W}\left(t_{f} ; a\right)=\hat{U}\left(t_{f}, t_{m}\right) \hat{W}\left(t_{m} ; a\right) \hat{U}^{\dagger}\left(t_{f}, t_{m}\right)$. The post-selection of the system can be described by positive operator-valued measure (POVM) $\hat{\pi}_{f}[33,34]$ which satisfies $\hat{\pi}_{f} \geq 0$ and $\sum_{f} \hat{\pi}_{f}=\hat{1}$, where $\hat{1}$ is an identity operator of the system and the summation is taken over all possible selections. 
Then, the joint probability $P(f, a)$ that the measurement yields the outcome $a$ and the post-selection $f$ is succeeded is given by $P(f, a)=\operatorname{Tr}_{E}\left[\left(\hat{\pi}_{f} \otimes \hat{1}_{E}\right) \hat{W}\left(t_{f} ; a\right)\right]$, where $\hat{1}_{E}$ stands for an identity operator of the environment. The posterior probability $P(a \mid f)$ that the measurement outcome $a$ is obtained under the condition that the post-selection $f$ is succeeded can be derived by making use of the Bayesian law [35], that is,

$P(a \mid f)=\frac{P(f, a)}{\sum_{a} P(f, a)}=\frac{\operatorname{Tr} \operatorname{Tr}_{E}\left[\left(\hat{\pi}_{f} \otimes \hat{1}_{E}\right) \hat{U}\left(t_{f}, t_{m}\right) \hat{W}\left(t_{m} ; a\right) \hat{U}^{\dagger}\left(t_{f}, t_{m}\right)\right]}{\sum_{a} \operatorname{Tr} \operatorname{Tr}_{E}\left[\left(\hat{\pi}_{f} \otimes \hat{1}_{E}\right) \hat{U}\left(t_{f}, t_{m}\right) \hat{W}\left(t_{m} ; a\right) \hat{U}^{\dagger}\left(t_{f}, t_{m}\right)\right]}$,

where the summation is taken over all possible measurement outcomes. Therefore, when the measurement is performed at the time $t_{m}$ between the pre-selection and the post-selection, the average value of the system observable $\hat{A}$ is calculated by $\bar{A}=\sum_{a} a P(a \mid f)$. To obtain the average value $\bar{A}$, we need to find the non-normalized postmeasurement state $\hat{W}\left(t_{m} ; a\right)$ of the system and environment.

To find the non-normalized state $\hat{W}\left(t_{m} ; a\right)$, we consider two kinds of measurement. One is strong measurement and the other is weak measurement. First, we suppose that strong measurement (or projective measurement) of an observable $\hat{A}$ is performed on the system at the measurement time $t_{m}$, which is described by a projection operator $|a\rangle\langle a|$, where $|a\rangle$ is an eigenstate of $\hat{A}$ such that $\hat{A}|a\rangle=a|a\rangle$. In this case, the non-normalized state $\hat{W}\left(t_{m}, a\right)$ just after the measurement is given by $\hat{W}\left(t_{m}, a\right)=|a\rangle\langle a| \otimes \hat{\rho}_{E}\left(t_{m} ; a\right)$ with the environmental operator $\hat{\rho}_{E}\left(t_{m} ; a\right)=\left\langle a\left|\hat{U}\left(t_{m}, t_{i}\right) \hat{W}_{i} \hat{U}^{\dagger}\left(t_{m}, t_{i}\right)\right| a\right\rangle$. Then, the posterior probability given by Eq. (1) becomes

$P(a \mid f)=\frac{\operatorname{Tr} \operatorname{Tr}_{E}\left[\left(\hat{\pi}_{f} \otimes \hat{1}_{E}\right) \hat{U}\left(t_{f}, t_{m}\right)\left[|a\rangle\langle a| \otimes \hat{\rho}_{E}\left(t_{m} ; a\right)\right] \hat{U}^{\dagger}\left(t_{f}, t_{m}\right)\right]}{\sum_{a} \operatorname{Tr}_{\operatorname{Tr}}\left[\left(\hat{\pi}_{f} \otimes \hat{1}_{E}\right) \hat{U}\left(t_{f}, t_{m}\right)\left[|a\rangle\langle a| \otimes \hat{\rho}_{E}\left(t_{m} ; a\right)\right] \hat{U}^{\dagger}\left(t_{f}, t_{m}\right)\right]}$,

which yields the strong value of the observable $A_{s}$,

$A_{s}=\frac{\sum_{a} a \operatorname{Tr} \operatorname{Tr}_{E}\left[\left(\hat{\pi}_{f} \otimes \hat{1}_{E}\right) \hat{U}\left(t_{f}, t_{m}\right)\left[|a\rangle\langle a| \otimes \hat{\rho}_{E}\left(t_{m} ; a\right)\right] \hat{U}^{\dagger}\left(t_{f}, t_{m}\right)\right]}{\sum_{a} \operatorname{Tr}_{\operatorname{Tr}}\left[\left(\hat{\pi}_{f} \otimes \hat{1}_{E}\right) \hat{U}\left(t_{f}, t_{m}\right)\left[|a\rangle\langle a| \otimes \hat{\rho}_{E}\left(t_{m} ; a\right)\right] \hat{U}^{\dagger}\left(t_{f}, t_{m}\right)\right]}$.

This result is valid for both non-Markovian and Markovian time evolution of the system.

In the rest of the paper, we confine ourselves to consider the case that the system obeys the Markovian time evolution which belongs to the dynamical semi-group [28-30]. The Markovian time evolution is valid if the environment has a very short correlation time and the coupling between the system and the environment is sufficiently weak. In this case, we can assume that the environment always stays in thermal equilibrium during the time evolution and we can approximate as $\hat{U}\left(t, t_{i}\right)\left(\hat{\rho}_{i} \otimes \hat{\rho}_{E}\right) \hat{U}^{\dagger}\left(t, t_{i}\right)=\mathrm{e}^{\left(t-t_{i}\right)} \hat{\mathscr{L}}_{\rho_{i}} \otimes \hat{\rho}_{E}$ [36], where the Lindblad operator $\hat{\mathscr{L}}$ [28-30] is given by

$\hat{\mathscr{L}} \hat{O}=-\frac{i}{\hbar}[\hat{H}, \hat{O}]+\sum_{\mu} \gamma_{\mu}\left(\hat{X}_{\mu} \hat{O} \hat{X}_{\mu}^{\dagger}-\frac{1}{2} \hat{X}_{\mu}^{\dagger} \hat{X}_{\mu} \hat{O}-\frac{1}{2} \hat{O} \hat{X}_{\mu}^{\dagger} \hat{X}_{\mu}\right)$

for any operator $\hat{O}$. In this equation, $\hat{H}$ is the Hamiltonian of the system, $X_{\mu}$ 's are a system operator and $\gamma_{\mu}$ 's are a relaxation parameter which is positive. Substituting $\hat{\rho}_{E}\left(t_{m} ; a\right)=\left\langle a\left|\mathrm{e}^{\left(t_{m}-t_{i}\right)} \hat{\mathscr{L}}_{\hat{\rho}}\right| a\right\rangle \hat{\rho}_{E}$ into Eq. (3), we obtain the strong value,

$A_{s}=\frac{\sum_{a} a \operatorname{Tr}^{\operatorname{Tr}}\left[\left(\hat{\pi}_{f} \otimes \hat{1}_{E}\right) \hat{U}\left(t_{f}, t_{m}\right)\left(|a\rangle\langle a| \otimes \hat{\rho}_{E}\right) \hat{U}^{\dagger}\left(t_{f}, t_{m}\right)\right]\left\langle a\left|\mathrm{e}^{\left(t_{m}-t_{i}\right)} \hat{\mathscr{L}} \hat{\rho}_{i}\right| a\right\rangle}{\sum_{a} \operatorname{Tr} \operatorname{Tr}_{E}\left[\left(\hat{\pi}_{f} \otimes \hat{1}_{E}\right) \hat{U}\left(t_{f}, t_{m}\right)\left(|a\rangle\langle a| \otimes \hat{\rho}_{E}\right) \hat{U}^{\dagger}\left(t_{f}, t_{m}\right)\right]\left\langle a\left|\mathrm{e}^{\left(t_{m}-t_{i}\right)} \hat{\mathscr{L}} \hat{\rho}_{i}\right| a\right\rangle}$. 
Since $\hat{U}\left(t_{f}, t_{m}\right)\left(|a\rangle\langle a| \otimes \hat{\rho}_{E}\right) \hat{U}^{\dagger}\left(t_{f}, t_{m}\right)=\mathrm{e}^{\left(t_{f}-t_{m}\right)} \hat{\mathscr{L}}|a\rangle\langle a| \otimes \hat{\rho}_{E}$ also holds in the Markovian time evolution, we finally obtain the strong value of the observable $\hat{A}$,

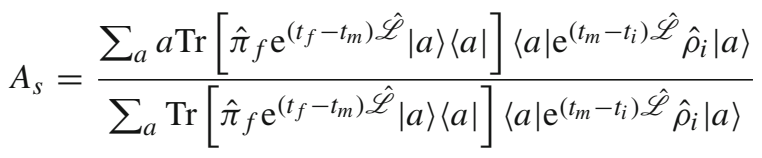

$$
\begin{aligned}
& =\frac{\sum_{a} a\left\langle a\left|\mathrm{e}^{\left(t_{f}-t_{m}\right) \hat{\mathscr{L}}^{\dagger}} \hat{\pi}_{f}\right| a\right\rangle\left\langle a\left|\mathrm{e}^{\left(t_{m}-t_{i}\right) \hat{\mathscr{L}}_{\hat{\rho}}} \hat{\rho}_{i}\right| a\right\rangle}{\sum_{a}\left\langle a\left|\mathrm{e}^{\left(t_{f}-t_{m}\right) \hat{\mathscr{L}}^{\dagger}} \hat{\pi}_{f}\right| a\right\rangle\left\langle a\left|\mathrm{e}^{\left(t_{m}-t_{i}\right) \hat{\mathscr{L}}} \hat{\rho}_{i}\right| a\right\rangle},
\end{aligned}
$$

where the conjugate of the Lindblad operator $\hat{\mathscr{L}}$ is defined by $\operatorname{Tr}\left[\left(\hat{\mathscr{L}}^{\dagger} \hat{O}\right) \hat{O}^{\prime}\right]=\operatorname{Tr}\left[\hat{O}\left(\hat{\mathscr{L}} \hat{O}^{\prime}\right)\right]$ for any operators $\hat{O}$ and $\hat{O}^{\prime}$, that is,

$\hat{\mathscr{L}}^{\dagger} \hat{O}=\frac{i}{\hbar}[\hat{H}, \hat{O}]+\sum_{\mu} \gamma_{\mu}\left(\hat{X}_{\mu}^{\dagger} \hat{O} \hat{X}_{\mu}-\frac{1}{2} \hat{X}_{\mu}^{\dagger} \hat{X}_{\mu} \hat{O}-\frac{1}{2} \hat{O} \hat{X}_{\mu}^{\dagger} \hat{X}_{\mu}\right)$.

The Lindblad operator always satisfies the equality $\hat{\mathscr{L}}^{\dagger} \hat{1}=0$ which is equivalent to the trace preservation. If the equality $\hat{\mathscr{L}} \hat{1}=\sum_{\mu} \gamma_{\mu}\left[\hat{X}_{\mu}, \hat{X}_{\mu}^{\dagger}\right]=0$ holds, the Lindblad operator $\hat{\mathscr{L}}$ is called unital. For example, the depolarizing channel and the dephasing channel are unital while the amplitude damping channel is not [37]. The intrinsic decoherence model proposed by Milburn [38] is also unital. This property plays an important role when we discuss the property of the linear response of the strong and weak values to an external field.

Next, we consider the case that the weak measurement of an observable $\hat{A}$ is performed at the measurement time $t_{m}$ between the pre-selection and the post-selection of the system. The weak measurement means that the interaction between the system and the measuring device is sufficiently weak and uncertainty of a pointer observable is very large in an initial device state [9]. The interaction between the system and the measuring device is assumed to be $\hat{H}_{\text {int }}=\hbar g \delta\left(t-t_{m}\right) \hat{A} \otimes \hat{P}_{M}[1-3]$, where $g$ is a coupling constant and $\hat{P}_{M}$ is a canonical conjugate of a pointer observable $\hat{Q}_{M}$ of the measuring device. Then, we find that the whole state of the relevant system, the environment and the measuring device just before the post-selection becomes

$$
\begin{aligned}
\hat{W}\left(t_{f}\right)= & \left(\hat{U}\left(t_{f}, t_{m}\right) \otimes \hat{1}_{M}\right)\left(\mathrm{e}^{-i g \hat{A} \otimes \hat{P}_{M}} \otimes \hat{1}_{E}\right) \\
& \times\left[\left(\hat{U}\left(t_{m}, t_{i}\right) \otimes \hat{1}_{M}\right)\left(\hat{\rho}_{i} \otimes \hat{\rho}_{E} \otimes \hat{\rho}_{M}\right)\left(\hat{U}^{\dagger}\left(t_{m}, t_{i}\right) \otimes \hat{1}_{M}\right)\right] \\
& \times\left(\mathrm{e}^{i g \hat{A} \otimes \hat{P}_{M}} \otimes \hat{1}_{E}\right)\left(\hat{U}^{\dagger}\left(t_{f}, t_{m}\right) \otimes \hat{1}_{M}\right),
\end{aligned}
$$

where $\hat{\rho}_{M}$ and $\hat{1}_{M}$ are an initial state and identity operator of the measuring device. The conditional state of the measuring device after the post-selection of the system is succeeded is given by

$\hat{\rho}_{M}^{f}=\frac{\operatorname{Tr}^{\operatorname{Tr}_{E}}\left[\left(\hat{\pi}_{f} \otimes \hat{1}_{E}\right) \hat{W}\left(t_{f}\right)\right]}{\operatorname{Tr}_{\operatorname{Tr}_{E}} \operatorname{Tr}_{M}\left[\left(\hat{\pi}_{f} \otimes \hat{1}_{E}\right) \hat{W}\left(t_{f}\right)\right]}$,

where $\operatorname{Tr}_{M}$ stands for the trace operation of the measuring device. The average shifts of the pointer observable and its canonical conjugate are calculated by $\Delta Q=\operatorname{Tr}_{M}\left[\hat{Q}_{M}\left(\hat{\rho}_{M}^{f}-\hat{\rho}_{M}\right)\right]$ and $\Delta P=\operatorname{Tr}_{M}\left[\hat{P}_{M}\left(\hat{\rho}_{M}^{f}-\hat{\rho}_{M}\right)\right]$. Taking the terms up to the first order with respect to the system-device coupling $g$, we can derive the real and imaginary parts of the weak value $A_{w}$ from $\Delta Q$ and $\Delta P$ [16]. The weak value $A_{w}$ of the observable $\hat{A}$ is given by

$A_{w}=\frac{\operatorname{Tr}_{\operatorname{Tr}}\left[\left(\hat{\pi}_{f} \otimes \hat{1}_{E}\right) \hat{U}\left(t_{f}, t_{m}\right) \hat{A} \hat{U}\left(t_{m}, t_{i}\right)\left(\hat{\rho}_{i} \otimes \hat{\rho}_{E}\right) \hat{U}^{\dagger}\left(t_{f}, t_{i}\right)\right]}{\operatorname{Tr}^{T_{r}}\left[\left(\hat{\pi}_{f} \otimes \hat{1}_{E}\right) \hat{U}\left(t_{f}, t_{i}\right)\left(\hat{\rho}_{i} \otimes \hat{\rho}_{E}\right) \hat{U}^{\dagger}\left(t_{f}, t_{i}\right)\right]}$, 
where we have used $\hat{U}\left(t_{f}, t_{i}\right)=\hat{U}\left(t_{f}, t_{m}\right) \hat{U}\left(t_{m}, t_{i}\right)$. Considering the Markovian time evolution [28-30], we obtain the weak value $A_{w}$,

$$
\begin{aligned}
A_{w} & =\frac{\operatorname{Tr}\left[\hat{\pi}_{f} \mathrm{e}^{\left(t_{f}-t_{m}\right) \hat{\mathscr{L}}} \hat{A} \mathrm{e}^{\left(t_{m}-t_{i}\right) \hat{\mathscr{L}}} \hat{\rho}_{i}\right]}{\operatorname{Tr}\left[\hat{\pi}_{f} \mathrm{e}^{\left(t_{f}-t_{i}\right) \hat{\mathscr{L}}} \hat{\rho}_{i}\right]} \\
& =\frac{\operatorname{Tr}\left[\left(\mathrm{e}^{\left(t_{f}-t_{m}\right) \hat{\mathscr{L}}^{\dagger}} \hat{\pi}_{f}\right) \hat{A}\left(\mathrm{e}^{\left(t_{m}-t_{i}\right)} \hat{\mathscr{L}}_{\rho_{i}}\right)\right]}{\operatorname{Tr}\left[\left(\mathrm{e}^{\left(t_{f}-t_{m}\right)} \hat{\mathscr{L}}^{\dagger} \hat{\pi}_{f}\right)\left(\mathrm{e}^{\left(t_{m}-t_{i}\right)} \hat{\mathscr{L}}_{\rho_{i}}\right)\right]} .
\end{aligned}
$$

The time dependence of the strong and weak values is determined by the two operators $\hat{\pi}_{f}\left(t_{m}\right)=\mathrm{e}^{\left(t_{f}-t_{m}\right) \hat{\mathscr{L}}^{\dagger}} \hat{\pi}_{f}$ and $\hat{\rho}_{i}\left(t_{m}\right)=\mathrm{e}^{\left(t_{m}-t_{i}\right) \hat{\mathscr{L}}} \hat{\rho}_{i}$. The latter is the usual time evolution that the quantum state evolves forwardly from the initial time $t_{i}$ to the measurement time $t_{m}$. The forward time evolution is determined by the Markovian quantum master equation of the Lindblad form, that is, $\partial \hat{\rho}_{i}(t) / \partial t=\hat{\mathscr{L}} \hat{\rho}_{i}(t)$. On the other hand, the POVM $\hat{\pi}_{f}$ is positive and can be regarded as a non-normalized state [39-42]. The non-normalized state $\hat{\pi}_{f}\left(t_{m}\right)=\mathrm{e}^{\left(t_{f}-t_{m}\right) \hat{\mathscr{L}}^{\dagger}} \hat{\pi}_{f}$ evolves from the post-selection time $t_{f}$ to the measurement time $t_{m}$ [36]. The backward time-evolution equation is determined by $\partial \pi_{f}(t) / \partial t=-\hat{\mathscr{L}}^{\dagger} \hat{\pi}_{f}(t)$. The normalized operator $\hat{\rho}_{f}(t)=\hat{\pi}_{f}(t) / \operatorname{Tr} \hat{\pi}_{f}(t)$ is referred to as the retrodictive state [39-42], time evolution of which is derived from the retrodictive quantum master equation $\partial \hat{\rho}_{f}(t) / \partial t=$ $-\hat{\mathscr{L}}^{\dagger} \hat{\rho}_{f}(t)+\left[\operatorname{Tr} \hat{\mathscr{L}}^{\dagger} \rho_{f}(t)\right] \hat{\rho}_{f}(t)$ [36]. In the following, we refer the normalized state $\hat{\rho}_{f}$ as the post-selected state and the initial state $\hat{\rho}_{i}$ as the pre-selected state. The post-selected state is also referred to as the retrodictive state [3942]. The pre-selected state obeys the forward (predictive) quantum master equation, the time-evolution generator of which is given by $\hat{\mathscr{L}}$. On the other hand, the post-selected state is subject to by the backward (retrodictive) quantum master equation [36], the time-evolution generator of which is given by $-\hat{\mathscr{L}}^{\dagger}$ if the equality $\hat{\mathscr{L}} \hat{1}=0$ holds.

The strong value $A_{s}$ and the weak value $A_{w}$ in the Markovian time evolution can be written as $A_{s}=\sum_{a} a P_{s}(a \mid f)$ and $A_{w}=\sum_{a} a P_{w}(a \mid f)$, where the strong and weak posterior probabilities are given, respectively, by

$$
\begin{aligned}
P_{s}(a \mid f) & =\frac{\left\langle a\left|\hat{\rho}_{f}\left(t_{m}\right)\right| a\right\rangle\left\langle a\left|\hat{\rho}_{i}\left(t_{m}\right)\right| a\right\rangle}{\sum_{a}\left\langle a\left|\hat{\rho}_{f}\left(t_{m}\right)\right| a\right\rangle\left\langle a\left|\hat{\rho}_{i}\left(t_{m}\right)\right| a\right\rangle}, \\
P_{w}(a \mid f) & =\frac{\sum_{a^{\prime}}\left\langle a^{\prime}\left|\hat{\rho}_{f}\left(t_{m}\right)\right| a\right\rangle\left\langle a\left|\hat{\rho}_{i}\left(t_{m}\right)\right| a^{\prime}\right\rangle}{\sum_{a, a^{\prime}}\left\langle a^{\prime}\left|\hat{\rho}_{f}\left(t_{m}\right)\right| a\right\rangle\left\langle a\left|\hat{\rho}_{i}\left(t_{m}\right)\right| a^{\prime}\right\rangle},
\end{aligned}
$$

which can be expressed $P_{s}(a \mid f)=\mathscr{P}_{s}\left[a \mid \hat{\rho}_{f}\left(t_{m}\right), \hat{\rho}_{i}\left(t_{m}\right)\right]$ and $P_{w}(a \mid f)=\mathscr{P}_{w}\left[a \mid \hat{\rho}_{f}\left(t_{m}\right), \hat{\rho}_{i}\left(t_{m}\right)\right]$. Here, we set

$$
\mathscr{P}_{S}[a \mid \hat{X}, \hat{Y}]=\frac{\langle a|\hat{X}| a\rangle\langle a|\hat{Y}| a\rangle}{\sum_{a}\langle a|\hat{X}| a\rangle\langle a|\hat{Y}| a\rangle}, \quad \mathscr{P}_{w}[a \mid \hat{X}, \hat{Y}]=\frac{\sum_{a^{\prime}}\left\langle a^{\prime}|\hat{X}| a\right\rangle\left\langle a|\hat{Y}| a^{\prime}\right\rangle}{\sum_{a, a^{\prime}}\left\langle a^{\prime}|\hat{X}| a\right\rangle\left\langle a|\hat{Y}| a^{\prime}\right\rangle} .
$$

The former satisfies the relation $\mathscr{P}_{s}[a \mid \hat{X}, \hat{Y}]=\mathscr{P}_{s}[a \mid \hat{Y}, \hat{X}]$ for any operators $\hat{X}$ and $\hat{Y}$ and the latter $\mathscr{P}_{w}[a \mid \hat{X}, \hat{Y}]=$ $\left(\mathscr{P}_{w}[a \mid \hat{Y}, \hat{X}]\right)^{*}$ for Hermitian operators $\hat{X}$ and $\hat{Y}$. In $P_{s}(a \mid f)$ and $P_{w}(a \mid f)$, the time evolution before the measurement at the time $t_{m}$ is separated from that after the measurement, since there is no correlation between the preand post-measurement states of the environment due to the Markovianity. If the environment is non-Markovian, the time evolutions before and after the measurement are correlated with each other due to the memory effect of the environment. Such correlation makes it very difficult to investigate the strong and weak values under the influence of the environment.

\section{Linear response of strong and weak values to an external field}

Using the results obtained in the previous section, we formulate a linear response theory for the strong value $A_{s}$ and the weak value $A_{w}$ in the Markovian quantum system when an weak $c$-number external field is applied to the 
system. We assume that the interaction Hamiltonian between the quantum system and the external field is given by $\hat{H}_{F}(t)=-\hbar F(t) \hat{B}$, where $F(t)$ is a $c$-number external field and $\hat{B}$ is a system observable coupled to the field [23]. In this case, the time-evolution super-operator of the system is given by

$$
\hat{\mathscr{U}}_{F}\left(t_{2}, t_{1}\right)=\mathrm{T} \exp \left[\int_{t_{1}}^{t_{2}} d t\left(\hat{\mathscr{L}}+i F(t) \hat{B}^{\times}\right)\right]
$$

where $\hat{B}^{\times} \hat{O}=[\hat{B}, \hat{O}]$ for any operator $\hat{O}$ and T stands for the time ordering that operators are placed from the right to the left in the chronological order. In the presence of the external field, the strong and weak values are obtained by replacing $\mathrm{e}^{\left(t_{2}-t_{1}\right) \hat{\mathscr{L}}}$ and $\mathrm{e}^{\left(t_{2}-t_{1}\right) \hat{\mathscr{L}}^{\dagger}}$ with $\hat{\mathscr{U}}_{F}\left(t_{2}, t_{1}\right)$ and $\hat{\mathscr{U}}_{F}^{\dagger}\left(t_{2}, t_{1}\right)$ in Eqs. (6) and (11) and thus we obtain

$$
\begin{aligned}
& A_{s}^{F}=\frac{\sum_{a} a\left\langle a\left|\hat{\mathscr{U}}_{F}^{\dagger}\left(t_{f}, t_{m}\right) \hat{\rho}_{f}\right| a\right\rangle\left\langle a\left|\hat{\mathscr{U}}_{F}\left(t_{m}, t_{i}\right) \hat{\rho}_{i}\right| a\right\rangle}{\sum_{a}\left\langle a\left|\hat{\mathscr{U}}_{F}^{\dagger}\left(t_{f}, t_{m}\right) \hat{\rho}_{f}\right| a\right\rangle\left\langle a\left|\hat{\mathscr{U}}_{F}\left(t_{m}, t_{i}\right) \hat{\rho}_{i}\right| a\right\rangle}, \\
& A_{w}^{F}=\frac{\operatorname{Tr}\left[\left(\hat{\mathscr{U}}_{F}^{\dagger}\left(t_{f}, t_{m}\right) \hat{\rho}_{f}\right) \hat{A}\left(\hat{\mathscr{U}}_{F}\left(t_{m}, t_{i}\right) \hat{\rho}_{i}\right)\right]}{\operatorname{Tr}\left[\left(\hat{\mathscr{U}}_{F}^{\dagger}\left(t_{f}, t_{m}\right) \hat{\rho}_{f}\right)\left(\hat{\mathscr{U}}_{F}\left(t_{m}, t_{i}\right) \hat{\rho}_{i}\right)\right]} .
\end{aligned}
$$

To investigate the linear response to the external field, we derive the differences $\Delta A_{s}=A_{s}^{F}-\left.A_{s}^{F}\right|_{F=0}$ and $\Delta A_{w}=A_{w}^{F}-\left.A_{w}^{F}\right|_{F=0}$ up to the first order with respect to the external field $F(t)$. Note that up to the first order, the time-evolution super-operator and its conjugate become

$$
\begin{aligned}
& \hat{\mathscr{U}}_{F}\left(t_{2}, t_{1}\right)=\mathrm{e}^{\left(t_{2}-t_{1}\right) \hat{\mathscr{L}}}+i \int_{t_{1}}^{t_{2}} d t \mathrm{e}^{\left(t_{2}-t\right) \hat{\mathscr{L}}} \hat{B}^{\times} \mathrm{e}^{\left(t-t_{1}\right) \hat{\mathscr{L}}} F(t) \\
& \hat{\mathscr{U}}_{F}^{\dagger}\left(t_{2}, t_{1}\right)=\mathrm{e}^{\left(t_{2}-t_{1}\right) \hat{\mathscr{L}}^{\dagger}}-i \int_{t_{1}}^{t_{2}} d t \mathrm{e}^{\left(t-t_{1}\right) \hat{\mathscr{L}}^{\dagger}} \hat{B}^{\times} \mathrm{e}^{\left(t_{2}-t\right) \hat{\mathscr{L}}^{\dagger}} F(t) .
\end{aligned}
$$

We first consider the linear response of the strong value $A_{s}$ to the external field $F(t)$. Substituting Eqs. (18) and (19) into (16) and neglecting all the terms higher than the first order with respect to the external field $F(t)$, we can derive

$$
\Delta A_{s}=\frac{\sum_{a}\left(a-A_{s}\right) X\left(t_{m}, a\right)\left\langle a\left|\hat{\rho}_{f}\left(t_{m}\right)\right| a\right\rangle}{\sum_{a}\left\langle a\left|\hat{\rho}_{f}\left(t_{m}\right)\right| a\right\rangle\left\langle a\left|\hat{\rho}_{i}\left(t_{m}\right)\right| a\right\rangle}+\frac{\sum_{a}\left(a-A_{s}\right) Y\left(t_{m}, a\right)\left\langle a\left|\hat{\rho}_{i}\left(t_{m}\right)\right| a\right\rangle}{\sum_{a}\left\langle a\left|\hat{\rho}_{f}\left(t_{m}\right)\right| a\right\rangle\left\langle a\left|\hat{\rho}_{i}\left(t_{m}\right)\right| a\right\rangle},
$$

with $\hat{\rho}_{i}\left(t_{m}\right)=\mathrm{e}^{\left(t_{m}-t_{i}\right) \hat{\mathscr{L}}} \hat{\rho}_{i}$ and $\hat{\rho}_{f}\left(t_{m}\right)=\mathrm{e}^{-\left(t_{m}-t_{f}\right) \hat{\mathscr{L}}^{\dagger}} \hat{\rho}_{f}$. In this equation, $X\left(t_{m}, a\right)$ and $Y\left(t_{m}, a\right)$ are given by

$$
\begin{aligned}
& X\left(t_{m}, a\right)=i \int_{t_{i}}^{t_{m}} d t\left\langle a\left|\mathrm{e}^{\left(t_{m}-t\right) \hat{\mathscr{L}}} \hat{B}^{\times} \mathrm{e}^{\left(t-t_{i}\right) \hat{\mathscr{L}}} \hat{\rho}_{i}\right| a\right\rangle F(t), \\
& Y\left(t_{m}, a\right)=-i \int_{t_{m}}^{t_{f}} d t\left\langle a\left|\mathrm{e}^{\left(t-t_{m}\right)} \hat{\mathscr{L}}^{\dagger} \hat{B}^{\times} \mathrm{e}^{\left(t_{f}-t\right) \hat{\mathscr{L}}^{\dagger}} \hat{\rho}_{f}\right| a\right\rangle F(t) .
\end{aligned}
$$

We can rewrite Eq. (20) into the following form:

$$
\Delta A_{s}=\int_{t_{i}}^{t_{m}} d t \Psi_{\rightarrow}^{A B}(t) F(t)+\int_{f_{f}}^{t_{m}} d t \Psi_{\leftarrow}^{A B}(t) F(t),
$$

where the forward and backward response functions for the strong value are given, respectively, by

$$
\begin{aligned}
& \Psi_{\rightarrow}^{A B}(t)=i \frac{\sum_{a}\left(a-A_{s}\right)\left\langle a\left|\hat{\rho}_{f}\left(t_{m}\right)\right| a\right\rangle\left\langle a\left|\mathrm{e}^{\left(t_{m}-t\right)} \hat{\mathscr{L}} \hat{B}^{\times} \mathrm{e}^{-\left(t_{m}-t\right) \hat{\mathscr{L}}} \hat{\rho}_{i}\left(t_{m}\right)\right| a\right\rangle}{\sum_{a}\left\langle a\left|\hat{\rho}_{f}\left(t_{m}\right)\right| a\right\rangle\left\langle a\left|\hat{\rho}_{i}\left(t_{m}\right)\right| a\right\rangle}, \\
& \Psi_{\leftarrow}^{A B}(t)=i \frac{\sum_{a}\left(a-A_{s}\right)\left\langle a\left|\mathrm{e}^{-\left(t_{m}-t\right) \hat{\mathscr{L}}^{\dagger}} \hat{B}^{\times} \mathrm{e}^{\left(t_{m}-t\right) \hat{\mathscr{L}}^{\dagger}} \hat{\rho}_{f}\left(t_{m}\right)\right| a\right\rangle\left\langle a\left|\hat{\rho}_{i}\left(t_{m}\right)\right| a\right\rangle}{\sum_{a}\left\langle a\left|\hat{\rho}_{f}\left(t_{m}\right)\right| a\right\rangle\left\langle a\left|\hat{\rho}_{i}\left(t_{m}\right)\right| a\right\rangle} .
\end{aligned}
$$


The first and second terms on the right-hand side of Eq. (23) represent the response of the strong value $A_{s}$ to the external field applied before and after the measurement of the observable $\hat{A}$. When the time evolution of the system is unitary, that is, $\hat{\mathscr{L}}=-\hat{\mathscr{L}}^{\dagger}=-(i / \hbar) \hat{H}^{\times}$, the result is equivalent to that obtained in the previous paper [27].

The forward and backward response functions can expressed as

$\Psi_{\rightarrow}^{A B}(t)=\mathscr{R}_{s}^{A B}\left[\hat{\rho}_{i}\left(t_{m}\right), \hat{\rho}_{f}\left(t_{m}\right) ; \hat{\mathscr{L}}\right]$,

$\Psi_{\leftarrow}^{A B}(t)=\mathscr{R}_{s}^{A B}\left[\hat{\rho}_{f}\left(t_{m}\right), \hat{\rho}_{i}\left(t_{m}\right) ;-\hat{\mathscr{L}}^{\dagger}\right]$,

with

$\mathscr{R}_{s}^{A B}[\hat{X}, \hat{Y} ; \hat{Z}]=i \frac{\sum_{a}\left(a-A_{s}\right)\left\langle a\left|\mathrm{e}^{\left(t_{m}-t\right) \hat{Z}} \hat{B}^{\times} \mathrm{e}^{-\left(t_{m}-t\right) \hat{Z}} \hat{X}\right| a\right\rangle\langle a|\hat{Y}| a\rangle}{\sum_{a}\langle a|\hat{X}| a\rangle\langle a|\hat{Y}| a\rangle}$.

From Eqs. (26) and (27), we find the symmetric property of the response functions. The backward response function $\Psi_{\leftarrow}^{A B}(t)$ is derived by the replacement $\hat{\rho}_{i}\left(t_{m}\right) \leftrightarrow \hat{\rho}_{f}\left(t_{m}\right)$ and $\hat{\mathscr{L}} \leftrightarrow-\hat{\mathscr{L}}^{\dagger}$ in the forward response function $\Psi_{\rightarrow}^{A B}(t)$ and vice versa. If the time evolution of the system is unitary, the equality $\hat{\mathscr{L}}=-\hat{\mathscr{L}}^{\dagger}$ holds.

We consider the case that the post-selection is not performed or any information is extracted from the system at the time $t_{f}$. This means that the post-selected state is given by $\hat{\rho}_{f}=\hat{1} / D$, where $D$ is a dimension of the Hilbert space of the system. Using the fact that an identity operator remains unchanged during the backward time evolution or equivalently $\hat{\mathscr{L}}^{\dagger} \hat{1}=0$, we can easily see that the backward response function becomes zero, that is, $\Psi_{\leftarrow}^{A B}(t)=0$. Thus, the external field applied after the measurement does not affect the strong value in the absence of the post-selection. By making use of $\hat{A}=\sum_{a} a|a\rangle\langle a|$ and $\sum_{a}\left\langle a\left|\mathrm{e}^{\left(t_{m}-t\right) \hat{\mathscr{L}}} \hat{B}^{\times} \mathrm{e}^{-\left(t_{m}-t\right) \hat{\mathscr{L}}} \hat{\rho}_{i}\left(t_{m}\right)\right| a\right\rangle=0$, the forward response function is given by

$\Psi_{\rightarrow}^{A B}(t)=\mathscr{R}_{s}^{A B}\left[\hat{\rho}_{i}\left(t_{m}\right), \hat{1} / D ; \hat{\mathscr{L}}\right]=i \operatorname{Tr}\left[\hat{A} \mathrm{e}^{\left(t_{m}-t\right) \hat{\mathscr{L}}} \hat{B}^{\times} \mathrm{e}^{-\left(t_{m}-t\right) \hat{\mathscr{L}}} \hat{\rho}_{i}\left(t_{m}\right)\right]$.

It is easy to see that if the time evolution is unitary, namely, $\hat{\mathscr{L}}=-(i / \hbar) \hat{H}^{\times}$, Eq. (29) becomes the usual response

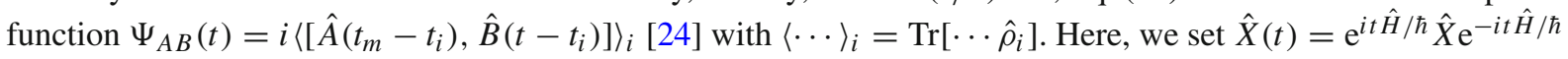
$(X=A, B)$.

Next, we suppose that we do not have any information on the initial state of the system. Then, the preselected state is given by $\hat{\rho}_{i}=\hat{1} / D$. It is important to note that the forward response function $\Psi_{\rightarrow}^{A B}(t)=$ $\mathscr{R}_{s}^{A B}\left[\mathrm{e}^{\left(t_{m}-t_{i}\right) \hat{\mathscr{L}}}(\hat{1} / D), \hat{\rho}_{f}\left(t_{m}\right) ; \hat{\mathscr{L}}\right]$ does not always vanish since in general the forward time evolution changes an identity operator, that is, $\hat{\mathscr{L}} \hat{1} \neq 0$. Here, let us assume that $\hat{\mathscr{L}}$ is unital $(\hat{\mathscr{L}} \hat{1}=0)$, which means that the trace of an operator is preserved during the backward time evolution. In this case, the forward response function becomes zero, that is, $\Psi_{\rightarrow}^{A B}(t)=0$. Furthermore, noting that $\hat{A}=\sum_{a} a|a\rangle\langle a|$ and $\sum_{a}\left\langle a\left|\mathrm{e}^{-\left(t_{m}-t\right) \hat{\mathscr{L}}^{\dagger}} \hat{B}^{\times} \mathrm{e}^{\left(t_{m}-t\right) \hat{\mathscr{L}}^{\dagger}} \hat{\pi}_{f}\left(t_{m}\right)\right| a\right\rangle=0$, we can derive the backward response function,

$\Psi_{\leftarrow}^{A B}(t)=\mathscr{R}_{s}^{A B}\left[\hat{\rho}_{f}\left(t_{m}\right), \hat{1} / D ;-\hat{\mathscr{L}}^{\dagger}\right]=i \operatorname{Tr}\left[\hat{A} \mathrm{e}^{-\left(t_{m}-t\right) \hat{\mathscr{L}}^{\dagger}} \hat{B}^{\times} \mathrm{e}^{\left(t_{m}-t\right) \hat{\mathscr{L}}^{\dagger}} \hat{\rho}_{f}\left(t_{m}\right)\right]$,

which becomes $\tilde{\Psi}_{A B}(t)=i\left\langle\left[\hat{A}\left(t_{m}-t_{f}\right), \hat{B}\left(t-t_{f}\right)\right]\right\rangle_{f}$ with $\langle\cdots\rangle_{f}=\operatorname{Tr}\left[\cdots \hat{\rho}_{f}\right]$ if the time evolution of the system is unitary. The result is summarized as follows:

\begin{tabular}{|c|c|c|}
\hline & $\hat{\rho}_{i}$ and $\hat{\rho}_{f}=\hat{1} / D$ & $\hat{\rho}_{i}=\hat{1} / D$ and $\hat{\rho}_{f}$ \\
\hline$\Psi_{\rightarrow}^{A B}(t)$ & $\mathscr{R}_{s}^{A B}\left[\hat{\rho}_{i}\left(t_{m}\right), \hat{1} / D ; \hat{\mathscr{L}}\right]$ & $\begin{array}{c}\mathscr{R}_{s}^{A B}\left[\mathrm{e}^{\left(t_{m}-t_{i}\right) \hat{\mathscr{L}}}(\hat{1} / D), \hat{\rho}_{f}\left(t_{m}\right) ; \hat{\mathscr{L}}\right] \\
(=0 \text { if } \hat{\mathscr{L}} \text { is unital })\end{array}$ \\
\hline$\Psi_{\leftarrow}^{A B}(t)$ & 0 & $\begin{array}{c}\mathscr{R}_{s}^{A B}\left[\hat{\rho}_{f}\left(t_{m}\right), \mathrm{e}^{\left.\left(t_{m}-t_{i}\right) \hat{\mathscr{L}}_{(}(\hat{1} / D) ;-\hat{\mathscr{L}}^{\dagger}\right]}\right. \\
\left(=\mathscr{R}_{s}^{A B}\left[\hat{\rho}_{f}\left(t_{m}\right), \hat{1} / D ;-\hat{\mathscr{L}}^{\dagger}\right] \text { if } \hat{\mathscr{L}} \text { is unital }\right)\end{array}$ \\
\hline
\end{tabular}


To find the linear response of the weak value to the external field, we substitute Eqs. (18) and (19) into Eq. (17) and neglect all the terms higher than the first order with respect to the external field. Then, we can drive

$\Delta A_{w}=\frac{\operatorname{Tr}\left[\hat{\rho}_{f}\left(t_{m}\right)\left(\hat{A}-A_{w}\right) \Delta \hat{\mathscr{U}}\left(t_{m}, t_{i}\right) \hat{\rho}_{i}\right]}{\operatorname{Tr}\left[\hat{\rho}\left(t_{m}\right) \hat{\rho}_{i}\left(t_{m}\right)\right]}+\frac{\operatorname{Tr}\left[\hat{\rho}_{f} \Delta \hat{\mathscr{U}}\left(t_{f}, t_{m}\right)\left(\hat{A}-A_{w}\right) \hat{\rho}_{i}\left(t_{m}\right)\right]}{\operatorname{Tr}\left[\hat{\rho}\left(t_{m}\right) \hat{\rho}_{i}\left(t_{m}\right)\right]}$,

where $\Delta \hat{\mathscr{U}}\left(t_{2}, t_{1}\right)$ is the second term on the right-hand side of Eq. (18). The numerators of the first and second terms on the right-hand side are

$$
\begin{aligned}
\operatorname{Tr} & {\left[\hat{\rho}_{f}\left(t_{m}\right)\left(\hat{A}-A_{w}\right) \Delta \hat{\mathscr{U}}\left(t_{m}, t_{i}\right) \hat{\rho}_{i}\right] } \\
& =i \int_{t_{i}}^{t_{m}} d t \operatorname{Tr}\left[\hat{\rho}_{f}\left(t_{m}\right)\left(\hat{A}-A_{w}\right) \mathrm{e}^{\left(t_{m}-t\right) \hat{\mathscr{L}}} \hat{B}^{\times} \mathrm{e}^{-\left(t_{m}-t\right) \hat{\mathscr{L}}} \hat{\rho}_{i}\left(t_{m}\right)\right] F(t), \\
\operatorname{Tr} & {\left[\hat{\rho}_{f} \Delta \hat{\mathscr{U}}\left(t_{f}, t_{m}\right)\left(\hat{A}-A_{w}\right) \hat{\rho}_{i}\left(t_{m}\right)\right] } \\
& =i \int_{t_{f}}^{t_{m}} d t i \operatorname{Tr}\left[\left(\mathrm{e}^{-\left(t_{m}-t\right) \hat{\mathscr{L}}^{\dagger}} \hat{B}^{\times} \mathrm{e}^{\left(t_{m}-t\right) \hat{\mathscr{L}}^{\dagger}} \hat{\rho}_{f}\left(t_{m}\right)\right)\left(\hat{A}-A_{w}\right) \hat{\rho}_{i}\left(t_{m}\right)\right] F(t) .
\end{aligned}
$$

Making use of the spectral decomposition $\hat{A}=\sum_{a} a|a\rangle\langle a|$ and the completeness relation $\sum_{a}|a\rangle\langle a|=\hat{1}$, we can rewrite Eq. (31) into the following form:

$\Delta A_{w}=\int_{t_{i}}^{t_{m}} d t \Phi_{\rightarrow}^{A B}(t) F(t)+\int_{t_{f}}^{t_{m}} d t \Phi_{\leftarrow}^{A B}(t) F(t)$,

where the forward and backward response functions for the weak value are given, respectively, by

$\Phi_{\rightarrow}^{A B}(t)=i \frac{\sum_{a, a^{\prime}}\left(a-A_{w}\right)\left\langle a^{\prime}\left|\hat{\rho}_{f}\left(t_{m}\right)\right| a\right\rangle\left\langle a\left|\mathrm{e}^{\left(t_{m}-t\right)} \hat{\mathscr{L}}_{B^{\times}} \times \mathrm{e}^{-\left(t_{m}-t\right) \hat{\mathscr{L}}} \hat{\rho}_{i}\left(t_{m}\right)\right| a^{\prime}\right\rangle}{\sum_{a, a^{\prime}}\left\langle a^{\prime}\left|\hat{\rho}_{f}\left(t_{m}\right)\right| a\right\rangle\left\langle a\left|\hat{\rho}_{i}\left(t_{m}\right)\right| a^{\prime}\right\rangle}$,
$\Phi_{\leftarrow}^{A B}(t)=i \frac{\sum_{a, a^{\prime}}\left(a-A_{w}\right)\left\langle a^{\prime}\left|\mathrm{e}^{-\left(t_{m}-t\right)} \hat{\mathscr{L}}^{\dagger} \hat{B}^{\times} \mathrm{e}^{\left(t_{m}-t\right)} \hat{\mathscr{L}}^{\dagger} \hat{\rho}_{f}\left(t_{m}\right)\right| a\right\rangle\left\langle a\left|\hat{\rho}_{i}\left(t_{m}\right)\right| a^{\prime}\right\rangle}{\sum_{a, a^{\prime}}\left\langle a^{\prime}\left|\hat{\rho}_{f}\left(t_{m}\right)\right| a\right\rangle\left\langle a\left|\hat{\rho}_{i}\left(t_{m}\right)\right| a^{\prime}\right\rangle}$.

If all the terms with $a \neq a^{\prime}$ in the summation can be neglected, these response functions become equal to Eqs. (24) and (25). When the system is subject to the unitary time evolution, this result is equal to that obtained in the previous paper [27]. Here, we introduce

$\mathscr{R}_{w}^{A B}[\hat{X}, \hat{Y} ; \hat{Z}]=i \frac{\sum_{a, a^{\prime}}\left(a-A_{w}\right)\left\langle a\left|\mathrm{e}^{\left(t_{m}-t\right) \hat{Z}} \hat{B}^{\times} \mathrm{e}^{-\left(t_{m}-t\right) \hat{Z}} \hat{X}\right| a^{\prime}\right\rangle\left\langle a^{\prime}|\hat{Y}| a\right\rangle}{\sum_{a, a^{\prime}}\left\langle a|\hat{X}| a^{\prime}\right\rangle\left\langle a^{\prime}|\hat{Y}| a\right\rangle}$,

in terms of which we can express the forward and backward response functions as

$\Phi_{\rightarrow}^{A B}(t)=\mathscr{R}_{w}^{A B}\left[\hat{\rho}_{i}\left(t_{m}\right), \hat{\rho}_{f}\left(t_{m}\right) ; \hat{\mathscr{L}}\right]$
$\Phi_{\leftarrow}^{A B}(t)=\left(\mathscr{R}_{w}^{A B}\left[\hat{\rho}_{f}\left(t_{m}\right), \hat{\rho}_{i}\left(t_{m}\right) ;-\hat{\mathscr{L}}^{\dagger}\right]\right)^{*}$.

It is easy to see that if the post-selection is not performed $\left(\hat{\rho}_{f}=\hat{1} / D\right)$, the forward response function $\Phi_{\rightarrow}^{A B}(t)$ is equal to Eq. (29) and backward response function becomes zero. On the other hand, if we have no information on the initial state $\left(\hat{\rho}_{i}=\hat{1} / D\right)$ and the equality $\hat{\mathscr{L}} \hat{1}=1$ holds, the forward response function vanishes and the backward response function $\Phi_{\leftarrow}^{A B}(t)$ becomes equal to Eq. (30). However, if $\hat{\mathscr{L}}$ is not unital, the forward response function $\Phi_{\rightarrow}^{A B}(t)$ does not vanish even in the absence of the pre-selection. The result is summarized as follows: 


\begin{tabular}{|c|c|c|}
\hline & $\hat{\rho}_{i}$ and $\hat{\rho}_{f}=\hat{1} / D$ & $\hat{\rho}_{i}=\hat{1} / D$ and $\hat{\rho}_{f}$ \\
\hline$\Phi_{\rightarrow}^{A B}(t)$ & $\begin{aligned} & \mathscr{R}_{w}^{A B}\left[\hat{\rho}_{i}\left(t_{m}\right), \hat{1} / D ; \hat{\mathscr{L}}\right] \\
= & \mathscr{R}_{s}^{A B}\left[\hat{\rho}_{i}\left(t_{m}\right), \hat{1} / D ; \hat{\mathscr{L}}\right]\end{aligned}$ & $\begin{array}{c}\mathscr{R}_{w}^{A B}\left[\mathrm{e}^{\left(t_{m}-t_{i}\right) \hat{\mathscr{L}}}(\hat{1} / D), \hat{\rho}_{f}\left(t_{m}\right) ; \hat{\mathscr{L}}\right] \\
(=0 \text { if } \hat{\mathscr{L}} \text { is unital })\end{array}$ \\
\hline$\Phi_{\leftarrow}^{A B}(t)$ & 0 & $\begin{aligned} & \mathscr{R}_{w}^{A B}\left[\hat{\rho}_{f}\left(t_{m}\right), \mathrm{e}^{\left(t_{m}-t_{i}\right)} \hat{\mathscr{L}}(\hat{1} / D) ;-\hat{\mathscr{L}}^{\dagger}\right]^{*} \\
(= & \left.\mathscr{R}_{s}^{A B}\left[\hat{\rho}_{f}\left(t_{m}\right), \hat{1} / D ;-\hat{\mathscr{L}}^{\dagger}\right] \text { if } \hat{\mathscr{L}} \text { isunital }\right)\end{aligned}$ \\
\hline
\end{tabular}

The forward time evolution of the pre-selected state $\hat{\rho}_{i}(t)$ is determined by the predictive quantum master equation $\partial \rho_{i}(t) / \partial t=\hat{\mathscr{L}} \hat{\rho}_{i}(t)$ [28-30] while the backward time evolution of the post-selected state $\hat{\rho}_{f}(t)=\hat{\pi}_{f}(t) / \operatorname{Tr} \hat{\pi}_{f}(t)$ is governed by the retrodictive quantum master equation $\partial \hat{\rho}_{f}(t) / \partial t=-\hat{\mathscr{L}}^{\dagger} \hat{\rho}_{f}(t)+\left[\operatorname{Tr}(\hat{\mathscr{L}} \hat{1}) \hat{\rho}_{f}(t)\right] \hat{\rho}_{f}(t)[36]$, the second term of which appears since $\hat{\mathscr{L}}^{\dagger}$ does not preserve the trace of an operator unless it is unital. The timeevolution generator $\hat{\mathscr{L}}$ always preserves the trace. If the equality $\hat{\mathscr{L}} \hat{1}=0$ holds, we find the symmetric property of the linear response theory within the framework of the ABL formalism.

\section{Linear response beyond strong and weak values}

In this section, using the method provided in Ref. [31], we extend the linear response theory to more general measurement that includes the strong and weak measurement as a special case. For this purpose, we expand the time-evolved pre-selected state $\hat{\rho}_{i}\left(t_{m}\right)$ just before the measurement in terms of the eigenstates of the measured observable $\hat{A}$ as $\hat{\rho}_{i}\left(t_{m}\right)=\sum_{a, a^{\prime}}|a\rangle\left\langle a\left|\hat{\rho}_{i}\left(t_{m}\right)\right| a^{\prime}\right\rangle\left\langle a^{\prime}\right|$. Furthermore, we assume that the interaction between the system and the measuring device induces the state change $|a\rangle \otimes|\phi\rangle \rightarrow|a\rangle \otimes\left|\phi_{a}\right\rangle$, where $|\phi\rangle$ is the initial state of the measuring device and $\left|\phi_{a}\right\rangle$ is a device state which depends on the value of the observable $\hat{A}$. Note that, if $\left|\phi_{a}\right\rangle$ 's are orthogonal with each other, it corresponds to the strong measurement while if $\left|\phi_{a}\right\rangle$ 's are almost indistinguishable from the initial state $|\phi\rangle$, it corresponds to the weak measurement. When the time evolution of the system is Markovian, we obtain the whole state of the system and the measuring device just before the post-selection,

$\hat{W}\left(t_{f}\right)=\left(\mathrm{e}^{\left(t_{f}-t_{m}\right) \hat{\mathscr{L}}} \otimes \hat{1}_{M}\right)\left[\sum_{a, a^{\prime}}\left\langle a\left|\mathrm{e}^{\left(t_{m}-t_{i}\right) \hat{\mathscr{L}}} \hat{\rho}_{i}\right| a^{\prime}\right\rangle|a\rangle\left\langle a^{\prime}|\otimes| \phi_{a}\right\rangle\left\langle\phi_{a^{\prime}}\right|\right]$.

Then after the post-selection of the system, the state of the measuring device becomes

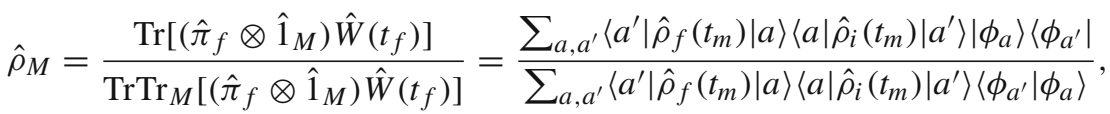

which yields the average value of a pointer observable $\hat{M}$ of the measuring device,

$\langle\hat{M}\rangle=\frac{\sum_{a, a^{\prime}}\left\langle a^{\prime}\left|\hat{\rho}_{f}\left(t_{m}\right)\right| a\right\rangle\left\langle a\left|\hat{\rho}_{i}\left(t_{m}\right)\right| a^{\prime}\right\rangle\left\langle\phi_{a^{\prime}}|\hat{M}| \phi_{a}\right\rangle}{\sum_{a, a^{\prime}}\left\langle a^{\prime}\left|\hat{\rho}_{f}\left(t_{m}\right)\right| a\right\rangle\left\langle a\left|\hat{\rho}_{i}\left(t_{m}\right)\right| a^{\prime}\right\rangle\left\langle\phi_{a^{\prime}} \mid \phi_{a}\right\rangle}$.

When $\left|\phi_{a}\right\rangle$ is an eigenstate of the pointer observable $\hat{M}$ such that $\hat{M}\left|\phi_{a}\right\rangle=m_{a}\left|\phi_{a}\right\rangle$ with eigenvalue $m_{a}=g a$, we can obtain the strong value of the system observable $\hat{A}$ since the equality $\langle\hat{M}\rangle=g A_{s}$ is established [31], where the strong value $A_{s}$ is given by Eq. (6). On the other hand, if all the states $\left|\phi_{a}\right\rangle$ are nearly equal to the initial state $|\phi\rangle$ and thus we can approximate as $\left\langle\phi_{a} \mid \phi_{a^{\prime}}\right\rangle \approx 1$, the average value (42) becomes

$\langle\hat{M}\rangle=\frac{\sum_{a, a^{\prime}}\left\langle a^{\prime}\left|\hat{\rho}_{f}\left(t_{m}\right)\right| a\right\rangle\left\langle a\left|\hat{\rho}_{i}\left(t_{m}\right)\right| a^{\prime}\right\rangle\left\langle\phi_{a^{\prime}}|\hat{M}| \phi_{a}\right\rangle}{\sum_{a, a^{\prime}}\left\langle a^{\prime}\left|\hat{\rho}_{f}\left(t_{m}\right)\right| a\right\rangle\left\langle a\left|\hat{\rho}_{i}\left(t_{m}\right)\right| a^{\prime}\right\rangle}$.

Here, we introduce two device observables $\hat{X}$ and $\hat{Y}$ which satisfy the relations $\left\langle\phi_{a^{\prime}}|\hat{X}| \phi_{a}\right\rangle=g\left(a^{\prime}+a\right) / 2$ and $\left\langle\phi_{a^{\prime}}|\hat{Y}| \phi_{a}\right\rangle=i g\left(a^{\prime}-a\right) / 2$. Then, setting $\hat{M}=\hat{X}+i \hat{Y}$, we can obtain the average value $\langle\hat{M}\rangle=g A_{w}$ with $A_{w}$ being given by Eq. (11), from which the weak value of the observable $\hat{A}$ is found [31]. Thus, we have found that 
the average value $\langle\hat{M}\rangle$ can yields the strong value and the weak value if we appropriately choose $\hat{M}$ and $\left|\phi_{a}\right\rangle$ of the measuring device.

Now, we investigate the linear response of the averaged pointer value $\langle\hat{M}\rangle$ to the external field $F(t)$. To drive the linear response, we replace $\hat{\rho}_{i}\left(t_{m}\right)=\mathrm{e}^{\left(t_{m}-t_{i}\right)} \hat{\mathscr{L}} \hat{\rho}_{i}$ and $\hat{\rho}_{f}\left(t_{m}\right)=\mathrm{e}^{-\left(t_{m}-t_{f}\right) \hat{\mathscr{L}}^{\dagger}} \hat{\rho}_{f}$ in Eq. (42) with $\hat{\mathscr{U}}_{F}\left(t_{m}, t_{i}\right) \hat{\rho}_{i}$ and $\hat{\mathscr{U}}_{F}^{\dagger}\left(t_{f}, t_{m}\right) \hat{\rho}_{f}$ and neglect all the terms higher than the first order with respect to the external field. After straightforward calculation, we can obtain the difference $\Delta M=\langle\hat{M}\rangle-\left.\langle\hat{M}\rangle\right|_{F=0}$ in the following form:

$\Delta M=\int_{t_{i}}^{t_{m}} d t \underset{\rightarrow}{M B}(t) F(t)+\int_{t_{f}}^{t_{m}} d t \underset{\leftarrow}{M B}(t) F(t)$,

where the forward response function $\underset{\Xi^{M B}}{\rightarrow}(t)$ and the backward response function $\underset{\Xi^{M B}}{\leftarrow}(t)$ of the pointer observable $\hat{M}$ are given, respectively, by

$\Xi_{\rightarrow}^{M B}(t)=i \frac{\sum_{a, a^{\prime}} M_{a^{\prime} a}\left\langle a^{\prime}\left|\hat{\rho}_{f}\left(t_{m}\right)\right| a\right\rangle\left\langle a\left|\mathrm{e}^{\left(t_{m}-t\right) \hat{\mathscr{L}}} \hat{B}^{\times} \mathrm{e}^{-\left(t_{m}-t\right) \hat{\mathscr{L}}} \hat{\rho}_{i}\left(t_{m}\right)\right| a^{\prime}\right\rangle}{\sum_{a, a^{\prime}}\left\langle a^{\prime}\left|\hat{\rho}_{f}\left(t_{m}\right)\right| a\right\rangle\left\langle a\left|\hat{\rho}_{i}\left(t_{m}\right)\right| a^{\prime}\right\rangle\left\langle\phi_{a^{\prime}} \mid \phi_{a}\right\rangle}$,
$\Xi_{\leftarrow}^{M B}(t)=i \frac{\sum_{a, a^{\prime}} M_{a^{\prime} a}\left\langle a^{\prime}\left|\mathrm{e}^{-\left(t_{m}-t\right) \hat{\mathscr{L}}^{\dagger}} \hat{B}^{\times} \mathrm{e}^{\left(t_{m}-t\right) \hat{\mathscr{L}}^{\dagger}} \hat{\rho}_{f}\left(t_{m}\right)\right| a\right\rangle\left\langle a\left|\hat{\rho}_{i}\left(t_{m}\right)\right| a^{\prime}\right\rangle}{\sum_{a, a^{\prime}}\left\langle a^{\prime}\left|\hat{\rho}_{f}\left(t_{m}\right)\right| a\right\rangle\left\langle a\left|\hat{\rho}_{i}\left(t_{m}\right)\right| a^{\prime}\right\rangle\left\langle\phi_{a^{\prime}} \mid \phi_{a}\right\rangle}$,

with $M_{a b}=\left\langle\phi_{a}|\hat{M}| \phi_{b}\right\rangle-\langle\hat{M}\rangle\left\langle\phi_{a} \mid \phi_{b}\right\rangle$. Furthermore, we can find the expression of the response functions,

$\Xi_{\rightarrow}^{M B}(t)=\mathscr{R}_{t}^{M B}\left[\hat{\rho}_{i}\left(t_{m}\right), \hat{\rho}_{f}\left(t_{m}\right) ; \hat{\mathscr{L}}\right]$,

$\Xi_{\leftarrow}^{M B}(t)=\left(\mathscr{R}_{t}^{M B}\left[\hat{\rho}_{f}\left(t_{m}\right), \hat{\rho}_{i}\left(t_{m}\right) ;-\hat{\mathscr{L}}^{\dagger}\right]\right)^{*}$,

with

$\mathscr{R}_{t}^{M B}[\hat{X}, \hat{Y} ; \hat{Z}]=i \frac{\sum_{a, a^{\prime}} M_{a^{\prime} a}\left\langle a\left|\mathrm{e}^{\left(t_{m}-t\right) \hat{Z}} \hat{B}^{\times} \mathrm{e}^{-\left(t_{m}-t\right) \hat{Z}} \hat{X}\right| a^{\prime}\right\rangle\left\langle a^{\prime}|\hat{Y}| a\right\rangle}{\sum_{a, a^{\prime}}\left\langle a|\hat{X}| a^{\prime}\right\rangle\left\langle a^{\prime}|\hat{Y}| a\right\rangle\left\langle\phi_{a^{\prime}} \mid \phi_{a}\right\rangle}$.

These results include the response functions for the strong value and the weak value as a special case. In fact, when $\left|\phi_{a}\right\rangle$ is the eigenstate of the pointer observable $\hat{M}$, Eqs. (45) and (46) reduce to Eqs. (24) and (25). On the other hand, when $\left|\phi_{a}\right\rangle \approx|\phi\rangle$ and $\hat{M}=\hat{X}+i \hat{Y}$, Eqs. (45) and (46) become Eqs. (35) and (36). If we do not perform the post-selection of the system and the post-selected state is $\hat{\rho}_{f}=\hat{1} / D$, the backward response function $\Xi_{\leftarrow}^{M B}(t)$ becomes zero and the forward response function $\Xi_{\rightarrow}^{M B}(t)$ is given by

$\Xi_{\rightarrow}^{M B}(t)=i \sum_{a}\left\langle\phi_{a}|\hat{M}| \phi_{a}\right\rangle\left\langle a\left|\mathrm{e}^{\left(t_{m}-t\right) \hat{\mathscr{L}}} \hat{B}^{\times} \mathrm{e}^{-\left(t_{m}-t\right) \hat{\mathscr{L}}} \hat{\rho}_{i}\left(t_{m}\right)\right| a\right\rangle$.

On the other hand, if we do not perform the pre-selection of the system $\left(\hat{\rho}_{i}=\hat{1} / D\right)$ and $\hat{\mathscr{L}}$ is unital $(\hat{\mathscr{L}} \hat{1}=0)$, the forward response function $\Xi_{\rightarrow}^{M B}(t)$ vanishes and the backward response function $\Xi_{\leftarrow}^{M B}(t)$ becomes

$\Xi_{\leftarrow}^{M B}(t)=i \sum_{a}\left\langle\phi_{a}|\hat{M}| \phi_{a}\right\rangle\left\langle a\left|\mathrm{e}^{-\left(t_{m}-t\right) \hat{\mathscr{L}}^{\dagger}} \hat{B}^{\times} \mathrm{e}^{\left(t_{m}-t\right) \hat{\mathscr{L}}^{\dagger}} \hat{\rho}_{f}\left(t_{m}\right)\right| a\right\rangle$.

Then, comparing this equation with Eq. (50), we can see that the backward (forward) response function is obtained from the forward (backward) response function by the exchange $\hat{\rho}_{i}\left(t_{m}\right) \leftrightarrow \hat{\rho}_{f}\left(t_{m}\right)$ and $\hat{\mathscr{L}} \leftrightarrow-\hat{\mathscr{L}}^{\dagger}$ if the time evolution generate $\hat{\mathscr{L}}$ is unital.

\section{Summary}

In this paper, we have formulated the linear response theory for the strong and weak values of an observable to an $c$ number external field within the framework of the ABL formalism. The measurement on the system is performed at the intermediate time between the pre-selection and the post-selection of the system. The Markovian time evolution 
has been assumed for the system which is placed under the influence of the environment, and thus the time evolution of the system is determined by the quantum master equation of the Lindblad form. Considering the change of the strong and weak values caused by the weak external field up to the first order, we have derived their linear response which is described in terms of the forward and backward response functions. The former (the latter) represents the effect of the external field on the measurement outcomes that is applied before (after) the measurement is performed. In the usual linear response theory without the post-selection, only the forward response function appears in the theory and the external field applied after the measurement does not affect the measurement outcomes. Considering the post-selection of the system, we have found the symmetric property of the linear response. Although the backward response function vanishes without the post-selection, the forward response function does not necessarily become zero even in the absence of the pre-selection since the time-evolution generator $\hat{\mathscr{L}}$ changes an identity operator unless it is unital. Furthermore, using the pointer observable of the measuring device, we have extended the linear response theory beyond the strong and weak values.

\section{References}

1. Von Neumann, J.: The Mathematical Foundations of Quantum Mechanics. Springer, Berlin (1932)

2. Peres, A.: Quantum Theory: Concepts and Methods. Kluwer, Dordrecht (1993)

3. Aharonov, Y., Rohrlich, D.: Quantum Paradoxes: Quantum Theory for the Perplexed. Wiley, Weinheim (2005)

4. Aharonov, Y., Bergmann, P.G., Lebowitz, J.L.: Time symmetry in the quantum process of measurement. Phys. Rev. 134, B1410B1416 (1964)

5. Aharonov, Y., Vaidman, L.: Complete description of a quantum system at a given time. J. Phys. A 24, 2315-2328 (1991)

6. Reznik, B., Aharonov, Y.: Time-symmetric formulation of quantum mechanics. Phys. Rev. 52, 2538-2550 (1995)

7. Vaidman, L.: On the two-state vector reformulation of quantum mechanics. Phys. Scr. T76, 95-92 (1998)

8. Aharonov, Y., Vaidman, L.: The two-vector state formalism: an updated review. In: Lecture Notes Physics, vol. 734, pp. $399-447$. Springer, Berlin (2008)

9. Aharonov, Y., Albert, D.Z., Vaidman, L.: How the result of a measurement of a component of the spin of a spin- $\frac{1}{2}$ particle can turn out to be 100. Phys. Rev. Lett. 60, 1351-1354 (1988)

10. Duck, M., Stevenson, P.M., Sudarshan, E.C.G.: The sense in which a “weak measurement" of a spin- $\frac{1}{2}$ particle's spin component yields a value 100. Phys. Rev. D 40, 2112-2217 (1989)

11. Aharonov, Y., Vaidman, L.: Properties of a quantum system during the time interval between two measurements. Phys. Rev. A 41, 11-20 (1990)

12. Wiseman, H.M.: Weak values, quantum trajectories, and the cavity-QED experiment on wave-particle correlation. Phys. Rev. A 65, 032111 (2002)

13. Johansen, L.M.: Weak measurements with arbitrary probe states. Phys. Rev. Lett. 93, 120402 (2004)

14. Johansen, L.M., Luis, A.: Nonclassicality in weak measurement. Phys. Rev. A 70, 052115 (2004)

15. Johansen, L.M.: What is the value of an observable between pre- and postselection. Phys. Lett. A 322, 298-300 (2004)

16. Jozsa, R.: Complex weak values in quantum measurement. Phys. Rev. A 76, 044103 (2007)

17. Lobo, A.C., Ribeiro, C.A.: Weak values and the quantum phase space. Phys. Rev. A 80, 012112 (2009)

18. Geszti, T.: Postselected weak measurement beyond the weak value. Phys. Rev. A 81, 044102 (2010)

19. Wu, S., Li, Y.: Weak measurements beyond the Aharonov-Albert-Vaidman formalism. Phys. Rev. A 83, 052106 (2011)

20. Zhu, X., Zhang, Y., Pang, S., Qiao, C., Liu, Q., Wu, S.: Quantum measurements with preselection and postselection. Phys. Rev. A 84, 052111 (2011)

21. Hofmann, H.F.: On the role of complex phases in the quantum statistics of weak measurements. New J. Phys. 13, 103009 (2011)

22. Kofman, A.G., Ashhab, S., Nori, F.: Nonperturbative theory of weak pre- and post-selected measurements. Phys. Rep. 520, 43-133 (2012)

23. Kubo, R.: Statistical-mechanical theory of irreversible process I: general theory and simple applications to magnetic and conduction problems. J. Phys. Soc. Jpn 12, 570-586 (1957)

24. Kubo, R., Tomita, M., Hashitsume, N.: Statistical Physics II. Springer, Berlin (1991)

25. Uchiyama, C., Aihara, M.: Role of initial quantum correlation in transient linear response. Phys. Rev. A 82, 044104 (2010)

26. Uchiyama, C.: Exploring initial correlations in a Gibbs state by application of external field. Phys. Rev. A 85, 052104 (2012)

27. Ban, M.: Linear response of a pre- and post-selected system to an external field. Phys. Lett. A 379, 284-288 (2015)

28. Breuer, H.-P., Petruccione, F.: The Theory of Open Quantum Systems. Oxford University Press, Oxford (2006)

29. Alicki, A., Lendi, K.: Quantum Dynamical Semigroups and Applications. Springer, Berlin (2007)

30. Rivas, A., Huelga, S.F.: Open Quantum Systems: An Introduction. Springer, Berlin (2012)

31. Salvail, J.Z.: Weak values beyond weak measurement. arXiv:1310.4193 [quant-ph]

32. Ban, M.: Weak values influenced by environment. J. Mod. Phys. 4(11A), 1-8 (2013) 
33. Helstrom, C.W.: Quantum Detection and Estimation Theory. Academic Press, New York (1977)

34. Holevo, A.S.: Probabilistic and Statistical Aspects of Quantum Theory. North-Holland, Amsterdam (1982)

35. Cover, T.M., Thomas, J.A.: Elements of Information Theory. Wiley, New York (1991)

36. Barnett, S.M., Pegg, D.T., Jeffers, J., Jedrkiewicz, O.: Mater equation for retrodiction of quantum communication signals. Phys. Rev. Lett. 86, 2455-2453 (2001)

37. Nielsen, M.A., Chuang, I.L.: Quantum Computation and Quantum Information. Cambridge University Press, Cambridge (2000)

38. Milburn, J.G.: Intrinsic decoherence in quantum mechanics. Phys. Rev. 44, 5401-5406 (1991)

39. Barnett, S.M., Pegg, D.T., Jeffers, J.: Bayes' theorem and quantum retrodiction. J. Mod. Opt. 47, 1779-1789 (2000)

40. Barnett, S.M., Pegg, D.T., Jeffers, J., Jedrkiewicz, O., Loudon, R.: Retrodiction for quantum optical communications. Phys. Rev. A 62, $022313(2000)$

41. Pegg, D.T., Barnett, S.M., Jeffers, J.: Quantum theory of preparation and measurement. J. Mod. Opt. 49, 913-924 (2002)

42. Pegg, D.T., Barnett, S.M., Jeffers, J.: Quantum retrodiction in open systems. Phys. Rev. A 66, 022106 (2002) 